Canadian

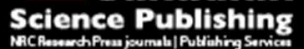

Canadian Journal of Physics

Revue canadienne de physique

\title{
Dc field microscopy of Rydberg Li atoms
}

\begin{tabular}{|r|l|}
\hline Journal: & Canadian Journal of Physics \\
\hline Manuscript ID & cjp-2015-0791.R2 \\
\hline Manuscript Type: & Article \\
\hline Complete List of Authors: & $\begin{array}{l}\text { Wang, De-hua; Ludong Universtiy } \\
\text { Cheng, Shaohao; Ludong University } \\
\text { Chen, Qiang; School of Physics and Optoelectronic Engineering, Ludong } \\
\text { University } \\
\text { Chen, Zhaohang; School of Physics and Optoelectronic Engineering, } \\
\text { Ludong University }\end{array}$ \\
\hline Keyword: & $\begin{array}{l}\text { photoionization, core scattering, Rydberg atom, microscopy, semiclassical } \\
\text { theory }\end{array}$ \\
\hline &
\end{tabular}

SCHOLARONE $^{\text {m }}$

Manuscripts 


\title{
De field microscopy of Rydberg Li atoms
}

\author{
Dehua Wang*,Shaohao Cheng, Qiang Chen and Zhaohang Chen \\ School of Physics and Optoelectronic Engineering, Ludong University, Yantai 264025, China
}

\begin{abstract}
The Dc field microscopy of Rydberg Li atoms has been studied on the basis of the semiclassical theory for the first time. Especially we discuss the atomic core scattering effect in the ionization dynamics of the Rydberg Li atom. Unlike the case of the photoionization of Rydberg $\mathrm{H}$ atom in an electric field, where the photoionization microscopy interference patterns are mainly caused by the Coulomb scattering and the electric field potential, for the photoionization of Rydberg Li atom in an electric field, the influence of the atomic core scattering effect on the photoionization microscopy interference patterns plays an important role. In addition, the structure of the interference pattern, which contains the spatial component of the electronic wave function, evolves smoothly with the electron energy above the saddle point energy. The observed oscillatory patterns in the electron probability density distributions on the detector plane are interpreted within the framework of the semiclassical approximation, which can be considered as a manifestation of interference between various electron trajectories arriving at a given point from the atom to the detector plane. This study provides some reference values for future experimental research on photoionization microscopy of the non-hydrogen Rydberg atoms in the presence of external fields.
\end{abstract}

Key Words: photoionization microscopy; core sacttering; semiclassical theory; Rydberg atom

PACS numbers: $32.80 . \mathrm{Fb}, 03.65 . \mathrm{Sq}, 07.81 .+\mathrm{a}$ 


\section{Introduction}

During the past several decades, the photodetachment or photoionization processes of negative ions or atoms in the external fields have been studied extensively both theoretically and experimentally[1-10]. The electron flux distribution has been measured using a position-sensitive detector placed at a macroscopic distance. Oscillatory structures appear in the electron flux distribution, which are caused from the electron waves travelling along different classical paths from the ion or atom to the detector. For the case of photodetachment of negative ion in an electric field, only two electron trajectories can arrive at a given point on the detector plane, which leads to a regular two-term interference pattern on the detector[11]. However, for the photoionization of atoms in an electric field, the ionized electron is affected both by the electric field and the long-range Coulomb field of the residual ion. The number of the electron trajectories becomes larger than in the case of the photodetachment, and an infinite number of electron trajectories can arrive at a given point on the detector plane[12-13]. Since hydrogen is a simple atom, which has only one electron, when the hydrogen atom is put in an electric field, the Stark Hamiltonian is exactly separable in terms of parabolic coordinates. For this reason, many researchers have studied the photoionization of hydrogen atom in the electric field [14-17]. The photoionization cross section and the positions and widths of resonances were calculated and found to be in excellent agreement with the experiment. In the early 1980s, Demkov and Kondratovich et al introduced the principle of photoionization microscopy theoretically for the first time [18-19]. They proposed an 
experimental method to study the ionization of hydrogen atom in an electric field. They suggested that after photoionization, the low-energy photoelectron waves can be projected onto a position-sensitive detector, which is oriented perpendicular to the electric field. An interference pattern on the detector will appear, which corresponds to the interference between electron trajectories evolving in the presence of the electric field and coulomb potential. This interference pattern can be split into two concentric structures, one is called direct interference if the electrons are barely interacting with the coulomb potential; another is called indirect interference if the electrons scatter on the coulomb potential. The "true" photoionization microscopy effect introduced by Kondratovich et al concerns a particular situation where the atom is ionized through a quasi-bound resonance in the ionization continuum. In that case the interference pattern observed corresponds to the square modulus of the wave function of the quasi bound state. This means that the pattern is a macroscopic projection of the atomic Stark wave function confined around the atom. The first experimental observation of the real "photoionization microscopy" effect was first published by Cohen et.al. They performed the photoionization microscopy experiments on $\mathrm{Li}$ atom in the presence of a static electric field[20]. The electron spatial distribution measured by their microscope is a direct macroscopic image of the projection of the microscopic squared modulus of the electron wave that is quasibound to the atom and constitutes the first experimental realization of the experiment proposed 30 years ago. The photoionization microscopy experiment was later confirmed in the case of $\mathrm{H}$ atoms in an electric field by Stodolna et al [21], who 
observed the nodal structure of the stark state wave function using the photoionization microscopy.

On the theoretical aspect, Zhao and Delos developed a semiclassical open orbit theory to study the dynamics of electron wave propagation in the photoionization microscopy of hydrogen atom in an electric field [22]. The open-orbit theory is based on the assumption that the electron waves propagate along different classical paths from a point-like source to a detector, provides a clear and intuitive physical picture to interpret structures of observed geometrical interference patterns in photoionization microscopy. Later, they used a quantum-mechanical method to calculate the spatial distributions of electron probability densities and current density for the photoionization of Rydberg hydrogen atom in an electric field[23]. The correspondence of the quantum-mechanical results with the semiclassical open orbit theory suggests the correctness of the semiclassical method. Ever since then, many researchers have used this theory to study the photoionization of hydrogen atom in other external fields, such as in the magnetic field, in parallel electric and magnetic fields, etc [24-27]. In these early studies, they all study the photoionization microscopy of Rydberg hydrogen atom in the external fields or environments. However, to our knowledge, the reports about the photoionization microscopy of alkali-metal Rydberg atom in external fields are relatively small. Since the properties of the alkali-metal Rydberg atoms are very similar to the Rydberg hydrogen atom, then what will happen for the photoionization of alkali-metal Rydberg atoms in external fields? In 1996, Lankhuijizen and Noordam studied the autoionization of a rubidium atom excited by a 
short laser pulse in a constant applied electric field [28-29]. They find the ionization of the Rydberg rubidium atom occurred via a train of electron pulses, rather than an exponential decay of the ionization of the hydrogen atom in an electric field. The pulses appeared in the rubidium were related to the core scattering effect. Later, Lin and his group have studied the autoionization of Rydberg lithium atoms in an electric field or in parallel electric and magnetic fields [30-32]. The above studies mainly study the ionization of Rydberg rubidium atom or Li atom in the external fields in the time domain. They calculated the variation of the ionization rate on the detector with the evolution time of the electron. As to the ionization of non-hydrogen Rydberg atom in the spatial domain, they didn't give a discussion. In this paper, we study the electron probability density distribution for the Rydberg Li atom in an electric field on the basis of the photoionization microscopy for the first time, especially we discuss the atomic core scattering effect on the photoionization microscopy interference patterns. Compared to the photoionization of hydrogen atom in an electric field, the electron is affected by a model potential of the residual ion, which leads to scattering by the atomic core. This model potential for the interaction of the active electron with the Li core was used previously by Main et al in the context of closed orbit theory for the study of the photoabsorption spectra of non-hydrogen Rydberg atom in a magnetic field[33-34]. Since the atomic core scattering potential cannot be neglected all the time, therefore the classical motion of the electron becomes much complicated. Not only the electron trajectories caused by the electric field and the Coulomb potential can arrive at the detector, some combination electron trajectories caused by the atomic 
scattering effect can also reach the detector, and these orbits play an important role in the electron probability density distribution on the detector plane. Regarding the role of the core-scattering, the effect has also been discussed by Cohen et.al. in a recent photoionization microscopy experiments on Li atom in a static electric field[20]. Our study provides a detailed explanation for the photoionization of Rydberg Li atom in the electric filed and can guide future researches on the photoionization of non-hydrogen atoms in external fields.

This paper is organized as follows: In section 2, we describe the photoionization process of the Rydberg Li atom in the presence of an electric field, and put forward an analytical formula for calculating the electron probability density distributions on the detector plane. In section 3, we calculate the electron probability density distributions on the detector plane, especially we analyze the atomic core scattering effect on the dynamics of the electron. The variation of the electron probability density distributions with the scaled energy above the classical saddle point energy is also analyzed in this part. Section 4 gives some conclusions of this paper. Scaled units are used unless other units are specified.

\section{Theory and quantitative formula}

The schematic plot of the photoionization of Li atom in an electric field is given in Fig.1. The electric field is along the $+\mathrm{z}$ axis and a detector plane is localized perpendicular to the $-\mathrm{z}$ axis. The $\mathrm{Li}$ atom lies at the origin of the coordinate system. The photoionization process can be regarded as a two step process: in the first step, after a ray of laser light polarized along z-axis irradiates on the Li atom, the valence 
electron in the Li atom may absorb a photon energy, then it is jumped into a high Rydberg state and generates an outgoing electron wave; in the second step, the electron wave propagates outward from the nucleus in all directions, following classical trajectory. As the electron moves far away from the nucleus, we can use the semiclassical approximation to construct the electron wave function [26]. The electrons traveling along the outgoing trajectories will escape from the atom, and the waves propagate to a large distance where a position-sensitive detector is placed. Due to the influence of the electric field and the modified Coulomb potential, more than two electron trajectories can reach the same point on the detector plane. The corresponding waves interfere constructively or destructively, which causes an observable interference pattern on the detector. This is called the photoionization microscopy. In the photoionization microscopy, the image formed by the electron wave on the detector is the square modulus of the transverse component of the electronic wave function, which can be measured using a velocity map imaging apparatus on the detector plane. A series of concentric interference fringes is schematically plotted on the detector plane as shown in Fig.1.

Using cylindrical coordinates $(\rho, \mathrm{z})$ and atomic units, the Hamiltonian for the Rydberg Li atom in an electric field is:

$$
H=\frac{1}{2}\left(p_{\rho}^{2}+p_{z}^{2}\right)+F z+V(\rho, z),
$$

where $F$ is the electric field. According to the radiation-transition selection rules, we choose the $z$ component of angular momentum $l_{z}=0 . V(\rho, z)$ is the modified Coulomb potential for the Rydberg Li atom, which includes the Coulomb potential plus the atomic core potential [30]: 
$V(\rho, z)=V_{\text {coulomb }}(\rho, z)+V_{\text {core }}(\rho, z)=-\frac{1}{\sqrt{\rho^{2}+z^{2}}}-\frac{z_{e}-1}{\sqrt{\rho^{2}+z^{2}}}\left(1+\frac{\sqrt{\rho^{2}+z^{2}}}{a}\right) e^{\frac{-\sqrt{\rho^{2}+z^{2}}}{a}}$,

where $r=\sqrt{\rho^{2}+z^{2}}, z_{e}=3$ is the atomic number and $a$ is a parameter, which is chosen to give the best agreement with the measured quantum defects. In the quantum mechanics, the atomic core potential introduces a phase shift in the calculation of the radial wave function that expresses itself as a quantum defect. Due to the influence of the atomic core potential, the principle quantum number becomes $n^{*}=n-\delta_{l} . \delta_{l}$ is the quantum defect, which depends on the angular momentum. The measured quantum defects for the $s$ and $p$ orbit of $\mathrm{Li}$ atom are $: \delta_{s}=0.4, \delta_{p}=0.05$. In our calculation, we take $a=1 / 2.13$ as used by Courtney et al for the calculation of the stark spectra of Li atom, which yields the quantum defects $\delta_{s}=0.4, \delta_{p}=0.06$ [35]. These values are very close to the measured quantum defects.

After transforming variables according to $\widetilde{\rho}=\rho F^{1 / 2}, \widetilde{z}=z F^{1 / 2}, \widetilde{a}=a F^{1 / 2}$, $\tilde{p}_{\rho}=p_{\rho} F^{-1 / 4}, \quad \tilde{p}_{z}=p_{z} F^{-1 / 4}, \tilde{t}=t F^{3 / 4}$ and $\varepsilon=E F^{-1 / 2}[30]$, we get the transformed Hamiltonian (the tilde “ " has been omitted):

$$
H=\frac{1}{2}\left(p_{\rho}^{2}+p_{z}^{2}\right)-\frac{1}{\sqrt{\rho^{2}+z^{2}}}-\frac{2}{\sqrt{\rho^{2}+z^{2}}}\left(1+\frac{\sqrt{\rho^{2}+z^{2}}}{a}\right) e^{\frac{-\sqrt{\rho^{2}+z^{2}}}{a}}+z=\varepsilon .
$$

From the above equation, we find that the scaled Hamiltonian does not depend upon the energy $E$ and electric field strength $F$ separately, but only on the scaled energy $\varepsilon$, thus eliminate a parameter.

The effective potential $V(\rho, z)$ in Eq.(3) has a saddle point, which is the root of the equations $\partial V(\rho, z) / \partial \rho=0$ and $\partial V(\rho, z) / \partial z=0$. The saddle point is critical for the calculation. Once the electron passes over the saddle point, it will be ionized and recorded by the detector eventually. Through careful calculation, we find the saddle point of the effective potential $V(\rho, z)$ is located at $\rho_{s}=0, z_{s}=-1.4834$. The 
corresponding saddle point energy is $\varepsilon_{s} \approx-2.396$. When the energy of the electron is bigger than the saddle point energy, the electron has access to the ionization channel through which the electron can be recorded by the detector. In this work, we place the detector at the plane $z=-4.0$.

In order to find the electron trajectories that reach the detector plane, we must integrate the Hamiltonian motion equations. From Eq.(3), we find there is a Coulomb singularity at the origin. Then we use the parabolic coordinates $(u, v)$ and their conjugate momenta $\left(p_{u}, p_{v}\right)$ instead of the cylindrical coordinates. The relation between the parabolic coordinates and the cylindrical coordinates is :

$$
\begin{aligned}
& \rho=u v, \quad z=\frac{1}{2}\left(u^{2}-v^{2}\right), r=\frac{1}{2}\left(u^{2}+v^{2}\right) \\
& p_{\rho}=\frac{v p_{u}+u p_{v}}{u^{2}+v^{2}}, \quad p_{z}=\frac{u p_{u}-v p_{v}}{u^{2}+v^{2}} .
\end{aligned}
$$

A new scaled time variable $\tau$ is defined by $d \tau / d t=1 /\left(u^{2}+v^{2}\right)$. After introducing an effective Hamiltonian $h=2 r(H-\varepsilon)$, we obtain :

$$
h=\frac{1}{2}\left(p_{u}^{2}+p_{v}^{2}\right)-\varepsilon\left(u^{2}+v^{2}\right)-4\left(1+\frac{u^{2}+v^{2}}{2 a}\right) e^{\frac{-\left(u^{2}+v^{2}\right)}{2 a}}+\frac{1}{2}\left(u^{4}-v^{4}\right)-2
$$

Let $h=0$, the Hamiltonian (Eq.(5)) can generate the same trajectories as setting $H=\varepsilon$. From the above equation, we find the Coulomb singularity has been removed, thus each electron trajectory can be launched from the origin. The outgoing angle of the electron between the initial velocity and the z-axis is $\theta$.

From the calculation, we find due to the influence of the electric field force and the modified Coulomb potential, more than one electron trajectory can arrive at a given point on the detector plane. Taking the scaled energy $\varepsilon=-0.5$ (scaled unit), through numerical calculation, we plot some of the electron trajectories that emitted from the origin and arrived at a given point on the detector plane. Fig.2(a) shows two different electron trajectories arriving at the same point $(0.43,-4.0)$ on the detector. 
The outgoing angles of the two electron trajectories are 2.986 and 0.732 , respectively. In Fig.2(b), three different electron trajectories can reach the same point $(0.0,-4.0)$ on the detector. Fig.2(c) shows four electron trajectories arriving at the same point $(1.03,-4.0)$ on the detector.

Next, we use the semiclassical approximation to construct the electron wave function. For the $j$-th electron trajectory, the corresponding wave function is denoted by $\psi_{j}\left(\rho, z_{0}\right)$, whose magnitude and phase depend on its classical density $A_{j}$ and a phase factor $\chi_{j}$ :

$$
\psi_{j}\left(\rho, z_{0}\right)=\sqrt{A_{j}\left(\rho, z_{0}\right)} \exp \left[i \chi_{j}\left(\rho, z_{0}\right)\right]
$$

Here, the classical density $A_{j}$ is given by[26]:

$$
A_{j}\left(\rho, z_{0}\right)=\frac{d \theta_{j}}{d \rho} \sin \theta_{j}
$$

where $\theta_{j}$ represents the outgoing angle for the $j$-th trajectory, the phase factor $\chi_{j}$ in Eq.(6) is:

$$
\chi_{j}=S_{j}(\rho, E) \omega-\frac{\pi}{2} \mu_{j} .
$$

where $\omega=F^{-1 / 4}, S_{j} \quad$ is the classical action: $S_{j}(\rho, E)=\int_{0}^{M\left(\rho, z_{0}\right)} \vec{p} \cdot d \vec{q} \cdot M\left(\rho, z_{0}\right)$ denotes a given point on the detector plane. $\mu_{j}$ is the Maslov index. In our calculation, the Maslov index includes not only the numbers of the maxima and minima of the cylindrical coordinates, but also includes the number when the classical density $A_{j}$ diverges[22].

For any given point on the detector plane, there exist several electron trajectories passing through it in the classically accessible region. Therefore, the final wave 
function $\psi_{f}\left(\rho, z_{0}\right)$ at point $M\left(\rho, z_{0}\right)$ can be obtained by summing over all the possible trajectories, leading from the origin to this point on the detector plane[26]:

$$
\begin{aligned}
\psi_{f}\left(\rho, z_{0}\right) & =\sum_{j=1}^{n} \psi_{j}\left(\rho, z_{0}\right) \\
& =\sum_{j=1}^{n}\left[\frac{d \theta_{j}}{d \rho} \sin \theta_{j}\right]^{1 / 2} \exp \left[i \chi_{j}\right]
\end{aligned} .
$$

The calculated radial electron probability density distribution at point $M\left(\rho, z_{0}\right)$ is given by the following formula:

$$
\begin{aligned}
\mathrm{P}\left(\rho, z_{0}\right) & =\left|\psi_{f}\left(\rho, z_{0}\right)\right|^{2} \\
& =\sum_{j=1}^{n} A_{j}+2 \sum_{i<j} \sqrt{A_{i} A_{j}} \cos \left(\chi_{i}-\chi_{j}\right)
\end{aligned}
$$

The first term is the classical probability density distribution, and the second term represents the interference among different classical trajectories arriving at a given point on the detector[24, 26].

\section{Results and Discussions}

By integrating the Hamiltonian motion equations (Eq.(5)) with the initial condition $\quad u(t=0)=0, \quad v(t=0)=0 \quad, \quad p_{u}(t=0)=\sqrt{12} \cos (\theta / 2)$, $p_{v}(t=0)=\sqrt{12} \sin (\theta / 2)$, we can search out all the ionization trajectories of the Li atom that can arrive at a given point on the detector plane. For different outgoing angle $\theta$, the evolution time $t$ that it takes for a trajectory from the electron source arrived at the detector plane is different. In this work, we stop the integration if the electron trajectory cannot reach the detector plane during a maximum period of time, $T_{\max }=30$ (scaled unit). For each electron trajectory, the outgoing angle $\theta$, the impact radius $\rho$ on the detector are recorded. Simultaneously, the classical action $S$ is calculated.

Firstly, we choose the scaled energy $\varepsilon=-0.5$, the Stark state with the principal 
quantum number $n=40$. Figure 3(a) plots a curve of the final position $\rho$ at the detector plane versus the outgoing angle $\theta$ of the electron for the Rydberg Li atom in an electric field. We find that the trajectory with the outgoing angle $\theta=\pi$ reaches the detector first. The electronic trajectories arrived at the detector can be divided into different types. The first kind is called the direct trajectory. The outgoing angle of the direct trajectories is usually large $(0.998<\theta \leq \pi)$. This type of electron trajectory heads directly towards the detector, after a short period of time, it reaches the detector without crossing the $\mathrm{z}$ axis. As $\theta$ decreases $(0<\theta \leq 0.998)$, the time it takes for an electron trajectory to arrive at the detector becomes longer. This kind of trajectory initially moves away from the z-axis, under the influence of the electric and the modified Coulomb potentials, it encircles the nucleus and finally hits the detector. This kind of orbit is called the indirect trajectory. For comparison, the $\rho$ - $\theta$ curve for the Rydberg $\mathrm{H}$ atom in an electric field is shown in Fig.3(b). By comparison the above two figures, we find a critical angle $\theta_{c}=0.5056$ is reached for the Rydberg $\mathrm{H}$ atom. All the electron trajectory with the outgoing angle less than $\theta_{c}$ cannot reach the detector within the given time. However, for the Rydberg Li atom, nearly all electron trajectories with the outgoing angle $0<\theta \leq \pi$ can strike the detector. As $0.5056<\theta \leq \pi$, the $\rho-\theta$ curve is similar to the case of $H$ atom. In the interval $0<\theta \leq 0.5056$, the $\rho-\theta$ curve becomes complicated and exhibits fractal structure as well as a certain self-similarity, which is similar to the epistrophic self-similarity structure when treating the escape-time dynamics in the ionization of atoms in external fields[36-37]. The spatial probability density distribution of electrons arising 
in photoionization microscopy of a Li atom in an electric field is shown in Fig.3(c), while the probability density distribution for the $\mathrm{H}$ atom is given in Fig.3(d). From these two figures, we find oscillatory structures all appear in the electron probability density distributions, which are caused by the interference effect of various electron trajectories arriving at a given point on the detector plane. However, for the Rydberg Li atom, the number of the ionization trajectories arriving at a given point on the detector increases, which causes the oscillatory structure in the electron probability density distributions to become much more complicated as compared to the case of Rydberg $\mathrm{H}$ atom. The difference between the electron probability density distributions for the $\mathrm{Li}$ atom and $\mathrm{H}$ atom can be qualitatively explained by analyzing the structure of the electron trajectories.

After the valence electron in the atom absorbs a photon from the laser pulse, it is promoted from a low-energy bound state into a high excited state in the form of an outgoing wave. This wave propagates away from the atom in all directions following classical trajectories. The classical electron dynamics is affected both by the electric field and the long-range modified Coulomb force of the residual ion. For a given scaled energy, the structure of the electron trajectory is related to the outgoing angle. After a period of time, some of the trajectories will escape the atom and arrive at the detector, making a contribution to the interference pattern in the electron probability density distributions. Other trajectories, however, may return to the nucleus, forming closed orbits [38]. For Rydberg $\mathrm{H}$ atom, the Coulomb scattering dominates. As the electron trajectories return to the vicinity of the atom, the waves associated with them 
are incoming Coulomb waves, which produce Coulomb scattered waves. The Coulomb scattered waves are strongly backwardly focused and retrace the orbit, thus generating repeated traversals of the trajectory[39]. Therefore, this type of trajectory will remain bound in the vicinity of the atom and have no contribution to the interference pattern in the electron probability density distributions. Some of this kind of electron trajectories are shown in Ref.[22] by Zhao and Delos. However, for the Rydberg $\mathrm{Li}$ atom, besides the Coulomb scattering, the atomic core-scattering also plays an important role. The atomic core scattering provides, in effect, a new electron wave source. As the electron trajectories return to the vicinity of the nucleus by the external electric field, the core scattering effect redistributes the amplitude of one closed orbit into some other ionizing orbits. Thus, a series of combination orbits appear, which can be considered as a combination of closed orbit with some other escaping trajectory[40]. Since the atomic core scattering induces important dynamical effects, it is now apparent that the contribution of the core-scattered trajectory in any semiclassical approach is necessary to be included. Some of the core-scattering trajectories are given in Fig.4.

For example, Fig.4(a) shows the electron trajectory emitted from the nucleus with the outgoing angle $\theta=0.326$. Under the influence of the electric field force plus the modified Coulomb force, the electron will return back to the nucleus and form a closed orbit. Due to the atomic core scattering effect, it will redistribute the amplitude of this closed orbit into another electron trajectories with the outgoing angle $\theta=1.685$, which is a direct electron trajectory. Therefore, the electron trajectory 
shown in Fig.4(a) can be split into a closed orbit plus an open orbit. Fig.4(b) can be considered as a combination of the closed orbit with the outgoing angle $\theta=0.496$ plus an open electron trajectory with the outgoing angle $\theta=1.106$. Similar description can be given to other combination orbits as plotted in Figs.4(c-f).

Figure 5 plots the interference patterns in the electron probability density distributions for the Rydberg Li atom in an electric field caused by different electron trajectories. They are obtained by calculating the electron probability density distributions through restricting the outgoing angle of the electron trajectory to a different region as shown in Fig.3(a). Fig.5(a) shows the electron probability density distributions with the outgoing angle of the electron trajectory limited in the region $0.5056<\theta \leq \pi$. Under this condition, the Coulomb scattering and the electric field force play an important role, therefore, the oscillatory structure in the electron probability density distributions curve is similar to the case of $\mathrm{H}$ atom which is shown in Fig.3(d). Fig.5(b) shows the electron probability density distributions with the outgoing angle of the electron trajectory limited in the interval $0<\theta \leq 0.5056$. In this region, the electron trajectories are a series of combination orbits induced by the atomic core scattering effect. We find oscillatory structures still appear in the electron probability density distributions, which suggests that the atomic core scattering effect in the electron probability density distributions on the detector plane cannot be neglected. In order to have a clear insight into the building up of the interference pattern, the total electron probability density is decomposed into contribution of the direct electron trajectories and the indirect electron trajectories. Fig. 5(c) shows the 
electron probability density caused by the direct electron trajectories, while Fig. 5(d) corresponds to the contribution of the indirect electron's trajectories to the electron probability density.

Next, we show how the photoionization microscopy interference patterns for the Rydberg Li atom in an electric field vary with the scaled energy. For this system, the saddle point energy $\varepsilon_{s} \approx-2.396$. As the excitation energy of the electron smaller than the saddle point energy, no electron trajectory can escape from the nucleus and reach the detector. In the following calculation, we choose the scaled energy varying from $\varepsilon=0.0$ down to $\varepsilon=-1.8$. Fig.6. shows the relation of the impact radius $\rho$ on the detector plane with the initial angle $\theta$ at which the electron trajectory is launched for different scaled energies. Fig.6(a) shows the $\rho$ - $\theta$ curve with the scaled energy $\varepsilon=0.0$. We find the whole $\rho-\theta$ curve is divided into several segments. For the electrons with the initial angle $0.661 \leq \theta \leq \pi$, they move towards the detector, after a short period of time, they will hit the detector without encircling the nucleus or crossing the $-\mathrm{z}$ axis. Under this condition, the electric field force plays a fundament role on the movement of the electron. These kind of trajectories are the direct trajectories[27]. As the initial outgoing angle $\theta<0.661$, both the electric field force and the modified Coulomb potential dominates, the electron will encircle the nucleus or cross the z-axis once or more times before it finally reach the detector. This family of trajectories belongs to the indirect trajectories. With the decrease of the scaled energy, the range of the outgoing angle for the direct trajectory becomes narrow. Besides, the maximum impact radius that the electron can reach decreases 
correspondingly. For example, at the scaled energy $\varepsilon=0.0$ (see Fig.6(a)), the maximum impact radius that the electron can reach $\rho_{\max }=4.465$. As the scaled energy is decreased to $\varepsilon=-1.8$, the maximum impact radius that the electron arrived at the detector is decreased to $\rho_{\max }=1.277$. The range of the direct electron trajectories is limited in a small region $2.26 \leq \theta \leq \pi$. Under this condition, the influence of the atomic core scattering effect plays the main role.

Finally, we calculate the electron probability density distribution on the detector plane for different scaled energies. The results are given in Figs.7-8. Fig.7 shows the variation of radial electron probability density distributions versus the impact radius $\rho$. From this figure, we find oscillatory structures appear in the electron probability density distributions, which can be considered as a consequence of the interference effect caused by different types of electron trajectories arriving at a given point on the detector plane. The top plot in Fig.7 shows the electron probability density distribution with the scaled energy $\varepsilon=0.0$. Under this circumstance, the oscillatory structures arise primarily from the interference of the direct electron trajectories, and a two-term interference pattern plays an important role. The electron probability distribution is widely spread out. With the decrease of the scaled energy, the number of the indirect electron trajectories becomes increased, which makes the interference pattern in the electron probability density distribution becomes complicated. In addition, as the scaled energy is decreased, the maximum impact radius that the electron can reach becomes decreased, which makes the oscillating region in the electron probability density distributions becoming reduced. For example, at the 
scaled energy $\varepsilon=-1.8$, the oscillatory structure is only limited in a small region $0<\rho<1.277$. In order to see the electron probability density distribution on the detector plane clearly, we plot the image plot of the electron probability density distributions for different scaled energies. The calculation results are shown in Fig.8. From this figure, we find at the same circular ring, the probability density distribution is the same. The electron probability density distribution on the detector plane consists of a series of interference rings. With the decrease of the scaled energy, the radius of the rings is decreased correspondingly. In addition, it is found that the electron probability density distribution on the detector plane consists of a series of bright and dark concentric rings. The bright rings correspond to the constructive interference of the electron's trajectories, while the dark rings correspond to the destructive interference. As the scaled energy is relatively large, $\varepsilon=-0.2$, the maximum impact radius on the detector is also large, which makes the radius of the outermost ring in the image plot becomes large, see Fig.8(a). The maximum impact radius on the detector becomes decreased with the decrease of the scaled energy, which makes the radius of the outermost ring in the image plot becomes small and the radius of the disk in the image plot gets reduced. See Figs.8(b-d).

\section{Conclusions}

We have performed a semiclassical calculation of the photoionization microscopy of Rydberg $\mathrm{Li}$ atom in an electric field, and have calculated the interference patterns in the photoelectron probability density distribution on a detector plane. The influence of the atomic core scattering effect on the electron dynamics has been discussed in great detail. Our study suggests that a lot of core-scattering 
combination ionization trajectories appear due to the atomic core scattering effect. Compared to the photoionization of the Rydberg $\mathrm{H}$ atom in an electric field, the number of the indirect electron trajectories arriving at the detector plane is increased, which makes the oscillatory patterns in the photoelectron probability density distribution become complicated. In addition, the oscillatory structures in the electron probability density distributions depend sensitively on the scaled energy. When the scaled energy is relatively small, the maximum impact radius that the electron can arrive at the detector plane is small, which means that the oscillating region in the probability density distributions is limited to a small region. When the scaled energy is close to the ionization threshold, the maximum impact radius that the electron can reach the detector plane becomes larger and the oscillating region in the probability density distributions becomes widely spread. Our calculation results further verify that the core scattering effect can play an important role in the photoionization for non-hydrogen Rydberg atoms. We hope that our results will be useful for guiding experimental studies of photoionization microscopy dynamics of non-hydrogen Rydberg atoms in external fields.

\section{Acknowledgements:}

This work is supported by the National Natural Science Foundation of China (Grant No. 11374133), a Project of Shandong Province Higher Educational Science and Technology Program of China (Grant No. J13LJ04), and Ph.D. Foundation of TangShan Normal University(Grant No. 2015A06). We also thank the referees for their good suggestions.

\section{References:}

[1] C. Blondel, C. Delsart, and F. Dulieu, Phys. Rev. Lett. 77, 3755(1996).

[2] C. Blondel, C. Delsart, F. Dulieu, and C. Valli, Eur. Phys. J. D5, 207 (1999). 
[3] C. Bordas, Phys. Rev. A 58, 400 (1998).

[4] F. Lepine, C. Bordas, C. Nicole, and M. J. J. Vrakking, Phys.Rev. A 70, 033417 (2004).

[5] C.Bracher, T.Kramer and J.B.Delos, Phys.Rev. A 73, 062114 (2006)..

[6] T. Kramer, C. Bracher, and M. Kleber, Europhys. Lett. 56, 471(2001).

[7] C. Nicole, I. Sluimer, F. Rosca-Pruna, M. Warntjes,M. Vrakking, C. Bordas, F. Texier, and F. Robicheaux, Phys.Rev. Lett. 85, 4024 (2000).

[8] T. Kramer, C. Bracher, and M. Kleber, J. Phys. A 35, 8361(2002).

[9] S. Gao, G. C. Yang, S. L. Lin, and M. L. Du, Eur. Phys. J. D 42,189 (2007).

[10]C.Bracher and J.B.Delos, Phys. Rev. Lett. 96, 100404(2006).

[11]M.L.Du, Phys.Rev. A 40, 4983 (1989).

[12] C. Nicole, H. L. Offerhaus, M. J. J. Vrakking,F. Lepine, and C. Bordas, Phys. Rev. Lett. 88, 133001(2002).

[13] C. Bordas, F. Lepine, C. Nicole, and M. J. J. Vrakking, Phys.Rev. A 68, 012709 (2003).

[14] L. D. Landau and E. M. Lifshitz, Quantum Mechanics:Non-Relativistic Theory, 3rd ed. (Elsevier, Amsterdam, 1981).

[15] R. J. Damburg and V. V. Kolosov, J. Phys. B 9, 3149 (1976).

[16] E. Luc-Koenig and A. Bachelier, J. Phys. B 13, 1743(1980).

[17] A. Alijah, J. T. Broad, and J. Hinze, J. Phys. B 25, 5043 (1992).

[18] Y. Demkov, V. Kondratovich and V. Ostrovskii, JETP Lett. 34, 403 (1982).

[19]V.D. Kondratovich and V.N. Ostrovsky, J. Phys. B 17,1981(1984);17, 2011(1984); 
23,3785(1990).

[20] S. Cohen, M.M. Harb, A. Ollagnier, F. Robicheaux, M.J.J. Vrakking, T. Barillot,

F. Lépine and C. Bordas, Phys. Rev. Lett. 110, 183001 (2013).

[21]A.S.Stodolna, A.Rouzee and F.Lepine et al, Phys. Rev. Lett., 110, 213001(2013).

[22] L.B. Zhao and J.B. Delos, Phys. Rev. A, 81, 053417(2010) .

[23] L.B. Zhao and J.B. Delos, Phys. Rev. A, 81, 053418(2010).

[24] D.H Wang, S.H.Cheng and Z.H.Chen, J.Electron.Spectrosc. 202,62 (2015).

[25] D.H Wang, T.T. Tang and L.J. Tian, Braz. J. Phys. 42,323(2012).

[26] L. Wang, H.F. Yang, X.J. Liu, H.P. Liu, M.S. Zhan and J.B. Delos, Phys. Rev. A, $82,022514(2010)$.

[27] H.F. Yang, L. Wang, X.J. Liu and H.P. Liu, Chin. Phys.B, 20, 063203(2011).

[28] G. M. Lankhuijzen and L. D. Noordam, Phys.Rev.Lett.76, 1784(1996).

[29] G. M. Lankhuijzen and L. D. Noordam, Phys.Rev.A 52, 2016(1995).

[30] H. Zhou, H. Y. Li, S. Gao, Y. H. Zhang, Z. M.Jia and S. L .Lin, Chin. Phys. B 17, 4428(2008).

[31] S. L. Lin, H. Zhou, X. Y. Xu, Z. M. Jia and S. H. Deng, Chin. Phys. Lett. 25, 4251(2008)

[32] S. H. Deng, S. Gao, Y. P. Li, X. Y. Xu, and S. L. Lin, Chin. Phys. B 19, 040511 (2010).

[33] B. Hüpper, J. Main, and Günter Wunner, Phys. Rev. Lett. 74, 2650 (1995).

[34] B. Hüpper, J. Main, and Günter Wunner, Phys . Rev. A 53, 744 (1996).

[35] M .Courtney, N. Spellmeyer, H. Jiao and D. Kleppner, Phys. Rev. A 51, 
3604(1995).

[36] K. A. Mitchell, J. P.Handley, B. Tighe and J. B. Delos, Phys. Rev. Lett. $92,073001(2004)$.

[37] K. A. Mitchell, J. P. Handley, B. Tighe and J. B. Delos, Phys. Rev. A 70, 043407(2004)

[38] M. L. Du and J. B. Delos, Phys. Rev. Lett. 58, 1731 (1987); M.L. Du and J. B. Delos, Phys. Rev. A 38, 1896 (1988); 38, 1913(1988).

[39] J. Gao and J. B. Delos, Phys. Rev. A 46, 1455(1992).

[40] D.H. Wang, Chin. Phys. B 20, 013403 (2011).

\section{Figure Captions:}

Fig.1. (Color online) Schematic description of photoionization process of $\mathrm{Li}$ atom in an electric field. The electric field $\mathrm{F}$ is along the $+\mathrm{z}$ axis and the detector plane is perpendicular to the $-\mathrm{Z}$ axis. The $\mathrm{Li}$ atom is placed at the origin of the coordinate system. When a laser light is irradiated on the $\mathrm{Li}$ atom, the electron wave propagates outward from the nucleus in all directions, following classical trajectories. If several trajectories reach the same point on the detector plane, interference pattern will appear in the electron probability density distribution. Here, we plot three electron trajectories reaching the same point on the detector plane, and a set of concentric interference fringes is schematically plotted.

Fig.2. (Color online) Some classical electron trajectories propagate from the nucleus and arrive at the same point on the detector. The scaled energy $\varepsilon=-0.5, n=80$, the electric filed strength $\mathrm{F}=125.54 \mathrm{~V} / \mathrm{cm}$. The detector is located at $\mathrm{z}=-4.0$ plane. 
Different trajectories are represented by different colors. The given point on the detector is as follows: (a) $\rho=0.43, z=-4.0$; (b) $\rho=0.0, z=-4.0$; (c) $\rho=1.03, z=-4.0$.

Fig.3(Color online). The $\rho-\theta$ curve and the electron probability density distribution on the detector plane with the scaled energy $\varepsilon=-0.5, n=80$, the electric filed strength $\mathrm{F}=125.54 \mathrm{~V} / \mathrm{cm}$. (a) The $\rho$ - $\theta$ curve for the Rydberg Li atom in an electric field. (b) The $\rho-\theta$ curve for the Rydberg $\mathrm{H}$ atom in an electric field. (c) The electron probability density distribution versus the impact radius $\rho$ for the Rydberg Li atom in an electric field. (d) The electron probability density distribution for the Rydberg $\mathrm{H}$ atom in an electric field. The detector is located at $\mathrm{z}=-4.0$ plane. The inset plots in Fig.3(c-d) are the image plot of the electron probability density distribution on the detector plane.

Fig.4 Some core-scattering combination ionizing trajectories of $\mathrm{Li}$ atom in an electric

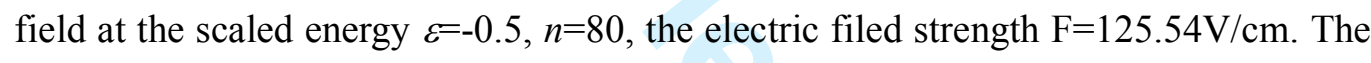
initial outgoing angle of the ionization trajectories are as follows: (a) $\theta=0.326$; (b) $\theta=0.496$; (c) $\theta=0.315$;(d) $\theta=0.446$; (e) $\theta=0.401$; (f) $\theta=0.076$.

Fig.5. The electron probability density distribution on the detector plane for the Rydberg Li atom in an electric field corresponding to different electron trajectories. The scaled energy $\varepsilon=-0.5, n=80$, the electric filed strength $F=125.54 \mathrm{~V} / \mathrm{cm}$. (a) The outgoing angle of the electron trajectory is limited in the region $0.5056<\theta \leq \pi$. (b) The outgoing angle of the electron trajectory belongs to the region $0<\theta \leq 0.5056$. Fig.6. Variation of the impact radius $\rho$ on the detector plane versus the outgoing angle of the electron at different scaled energies. The detector is located at $\mathrm{z}=-4.0$ plane. The scaled energy is given in each plot.

Fig.7. Variation of the electron probability density distribution on the detector plane 
versus the impact radius $\rho$ at different scaled energies. The detector is located at $\mathrm{z}=-4.0$ plane. The scaled energy is given in each plot.

Fig.8. (Color online) The three dimensional electron probability density distribution on the detector plane at different scaled energies. The detector is located at $\mathrm{z}=-4.0$ plane. The scaled energy is given in each plot. The left column corresponds to the three dimensional probability density distributions on the detector plane, while the right column shows the corresponding image plots.

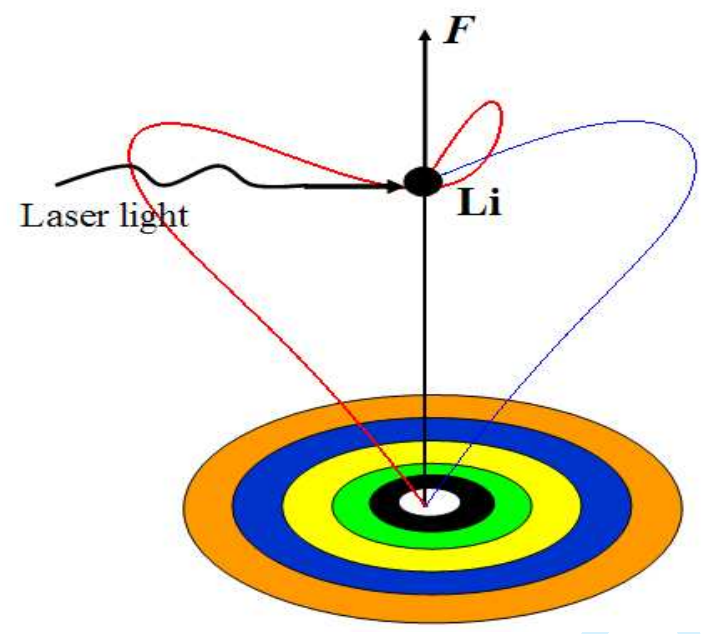

Fig.1. (Color online) Schematic description of photoionization process of Li atom in an electric field. The electric field $F$ is along the $+\mathrm{z}$ axis and the detector plane is perpendicular to the $-\mathrm{z}$ axis. The $\mathrm{Li}$ atom is placed at the origin of the coordinate system. When a laser light is irradiated on the Li atom, the electron wave propagates outward from the nucleus in all directions, following classical trajectories. If several trajectories reach the same point on the detector plane, interference pattern will appear in the electron probability density distribution. Here, we plot three electron trajectories reaching the same point on the detector plane, and a set of concentric interference fringes is schematically plotted. 

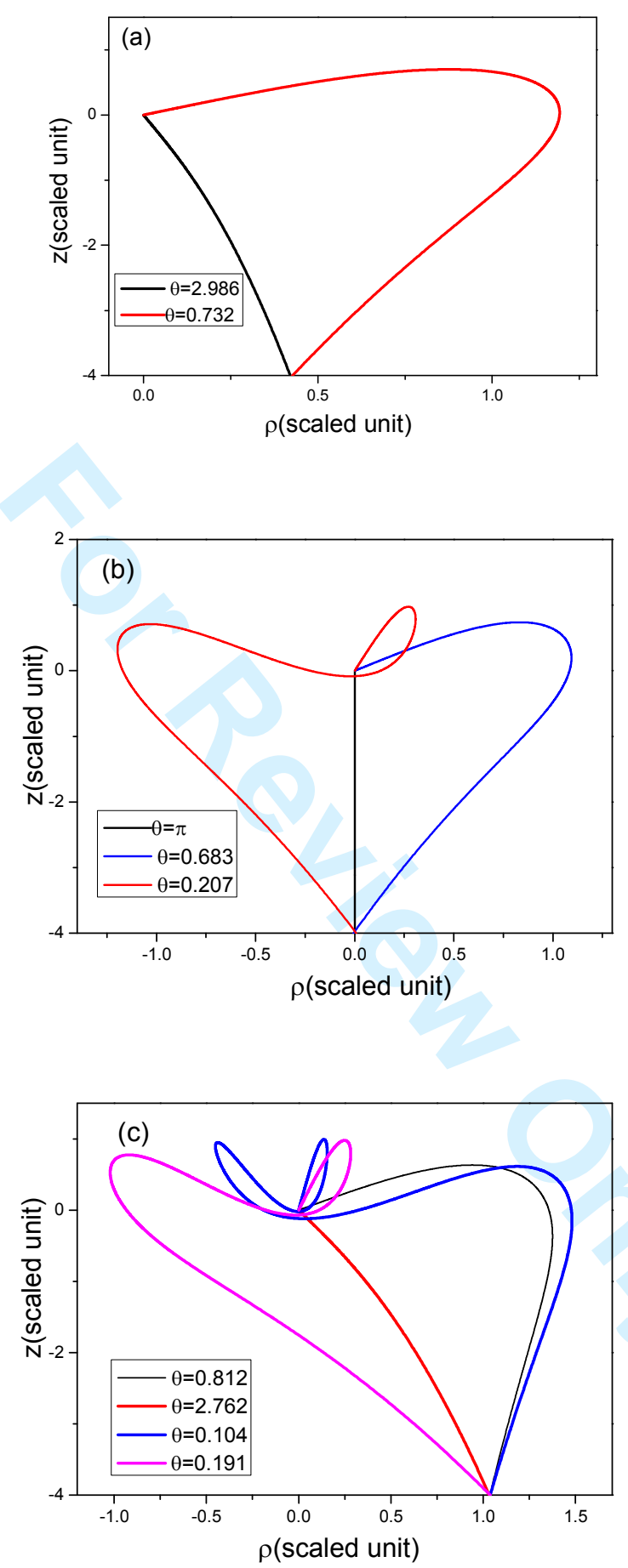

Fig.2. (Color online) Some classical electron trajectories propagate from the nucleus and arrive at the same point on the detector. The scaled energy $\varepsilon=-0.5$. The detector is located at $z=-4.0$ plane. Different trajectories are represented by different colors. The 
given point on the detector is as follows: (a) $\rho=0.43, z=-4.0$; (b) $\rho=0.0, z=-4.0$; (c) $\rho=1.03, z=-4.0$.
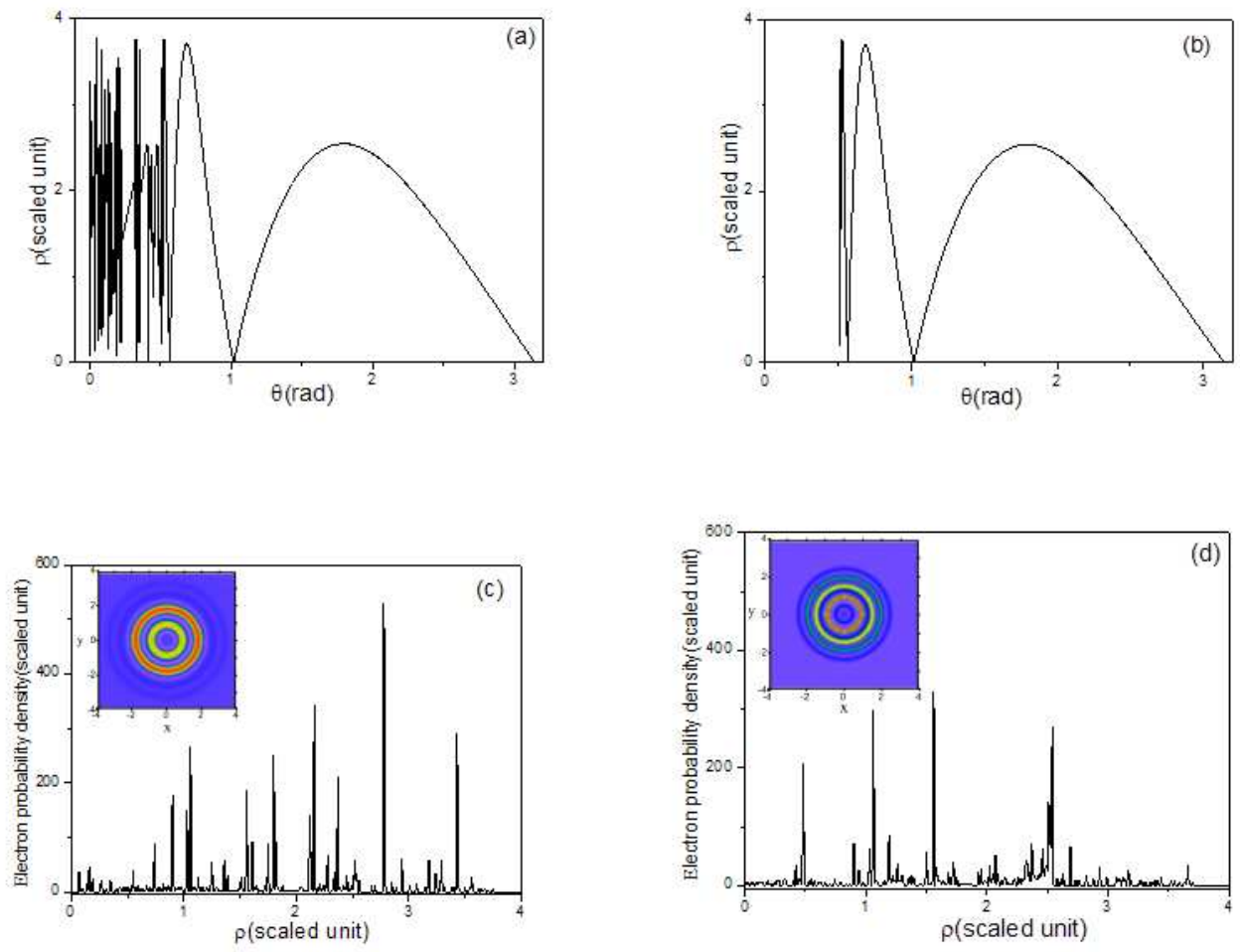

Fig.3(Color online). The $\rho$ - $\theta$ curve and the electron probability density distribution on the detector plane with the scaled energy $\varepsilon=-0.5$. (a) The $\rho-\theta$ curve for the Rydberg Li atom in an electric field. (b) The $\rho$ - $\theta$ curve for the Rydberg $\mathrm{H}$ atom in an electric field. (c) The electron probability density distribution versus the impact radius $\rho$ for the Rydberg Li atom in an electric field. (d) The electron probability density distribution for the Rydberg $\mathrm{H}$ atom in an electric field. The detector is located at $\mathrm{z}=-4.0$ plane. The inset plots in Fig.3(c-d) are the image plot of the electron probability density distribution on the detector plane. 

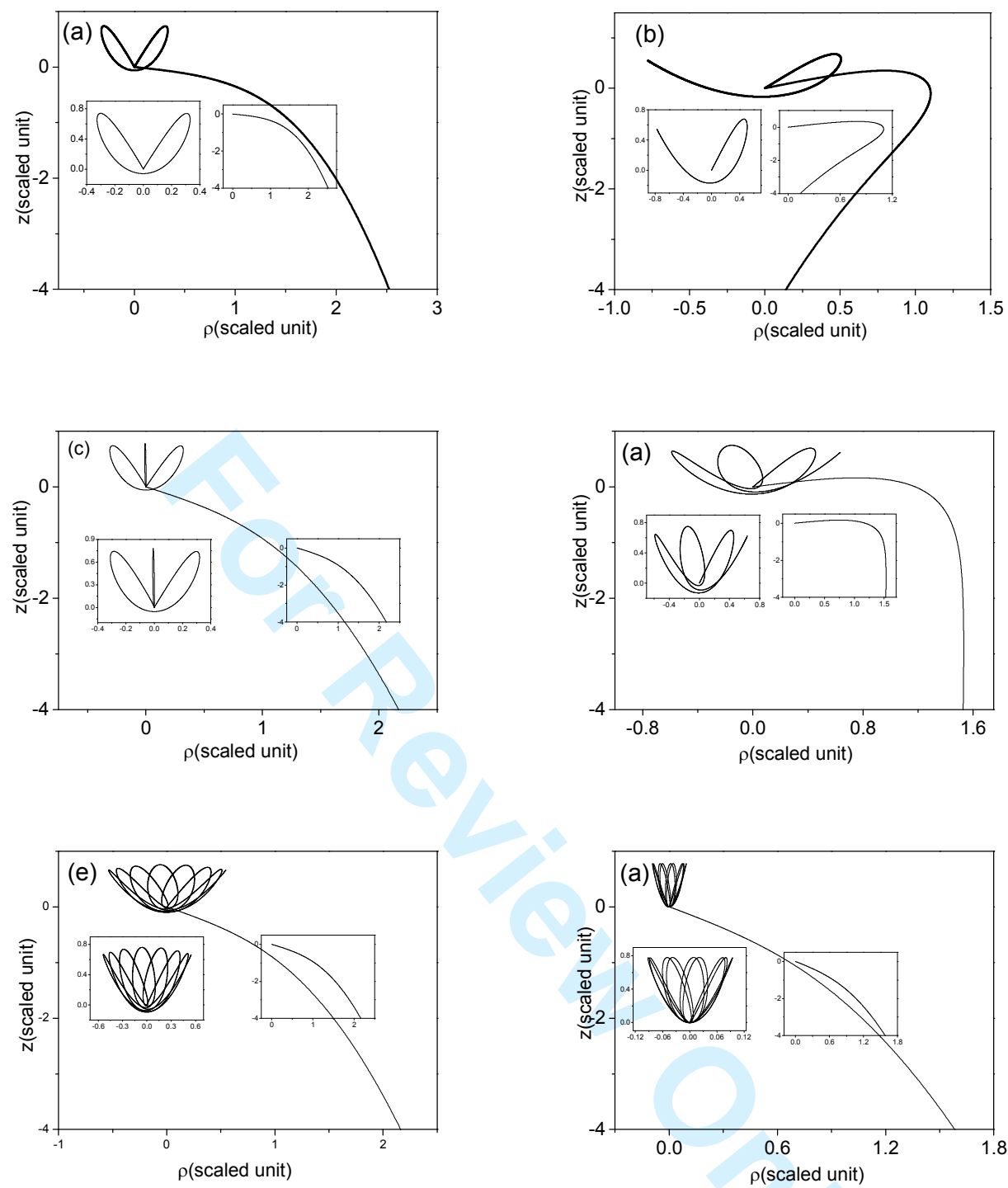

Fig.4 Some core-scattering combination ionizing trajectories of Li atom in an electric field at the scaled energy $\varepsilon=-0.5$. The initial outgoing angle of the ionization trajectories are as follows: (a) $\theta=0.326$; (b) $\theta=0.496$; (c) $\theta=0.315$;(d) $\theta=0.446$; (e) $\theta=0.401$; (f) $\theta=0.076$. 

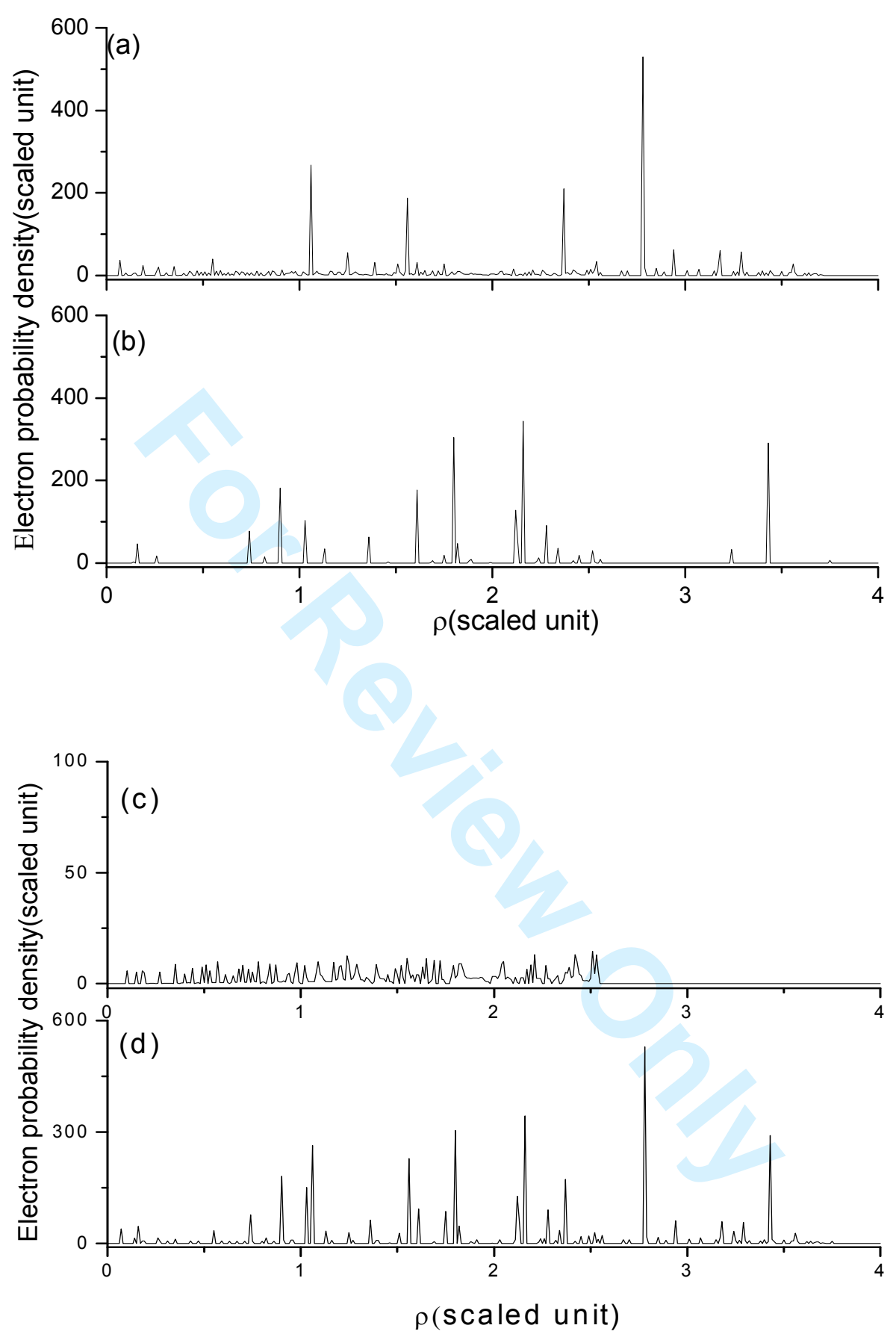

Fig.5. The electron probability density distribution on the detector plane for the Rydberg Li atom in an electric field corresponding to different electron trajectories. The scaled energy $\varepsilon=-0.5$. (a) The outgoing angle of the electron trajectory is limited in the region $0.5056<\theta \leq \pi$. (b) The outgoing angle of the electron trajectory 
belongs to the region $0<\theta \leq 0.5056$. (c) The electron probability density caused by the direct electron trajectories. (d) The electron probability density caused by the indirect electron trajectories.
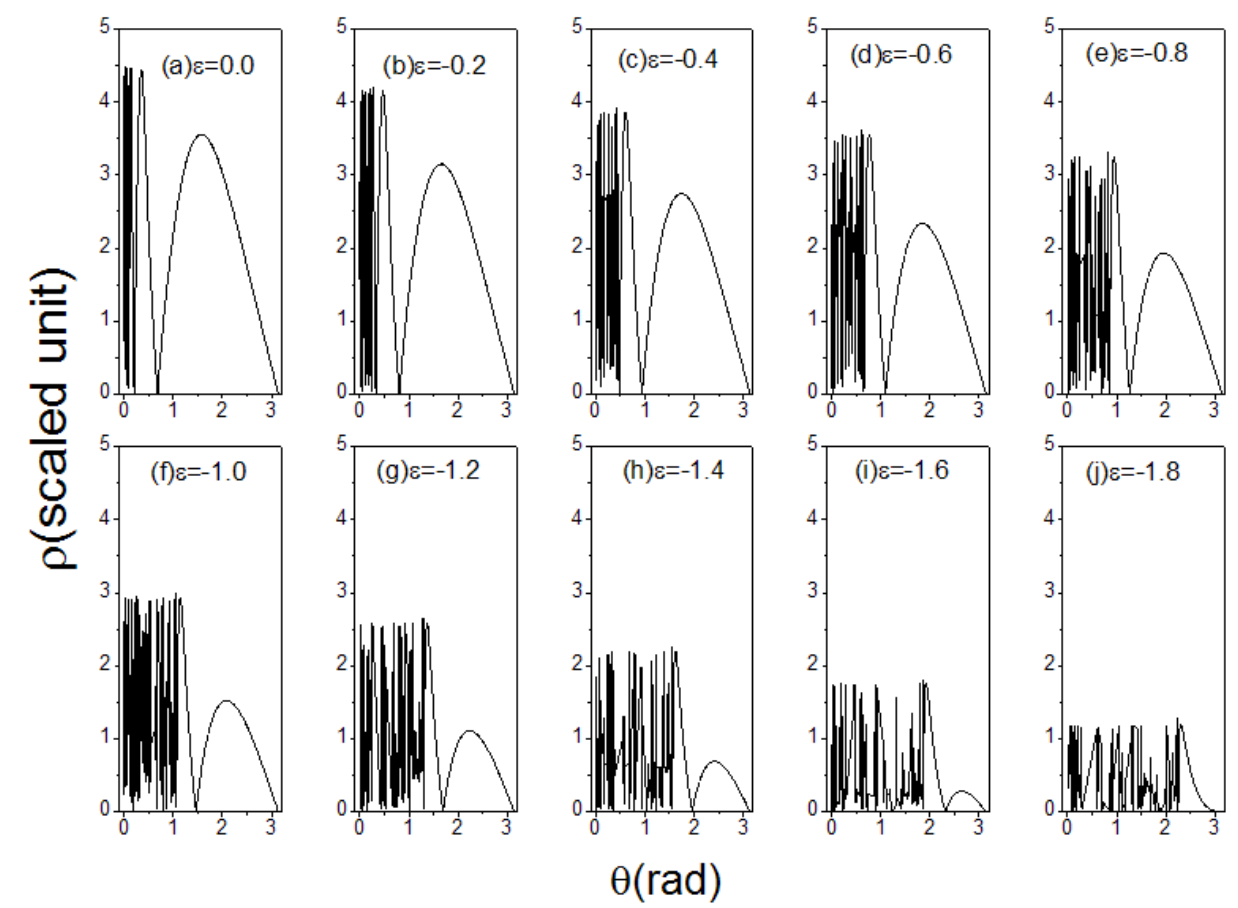

Fig.6. Variation of the impact radius $\rho$ on the detector plane versus the outgoing angle of the electron at different scaled energies. The detector is located at $\mathrm{z}=-4.0$ plane. The scaled energy is given in each plot. 


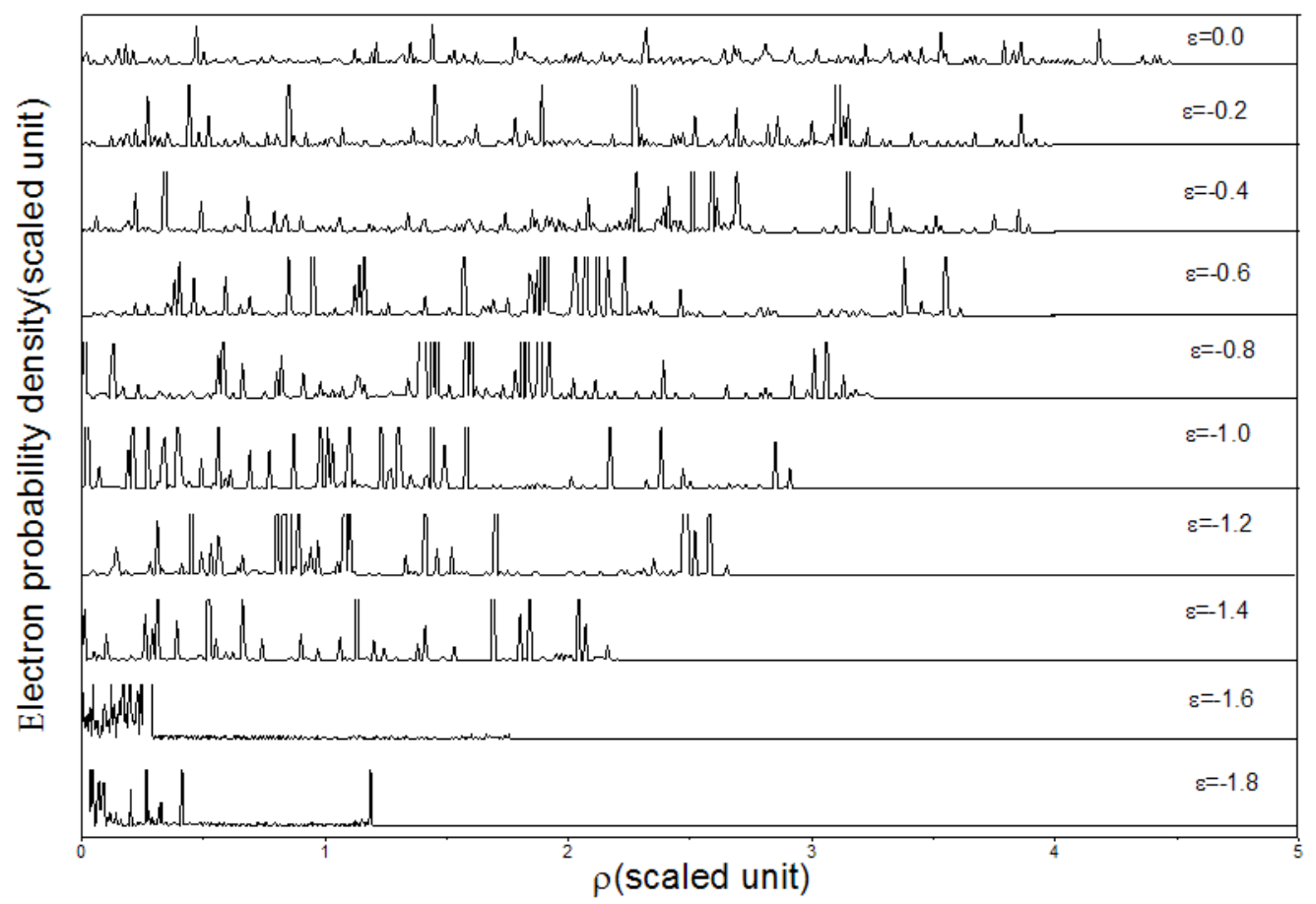

Fig.7. Variation of the electron probability density distribution on the detector plane versus the impact radius $\rho$ at different scaled energies. The detector is located at $\mathrm{Z}=-4.0$ plane. The scaled energy is given in each plot. 


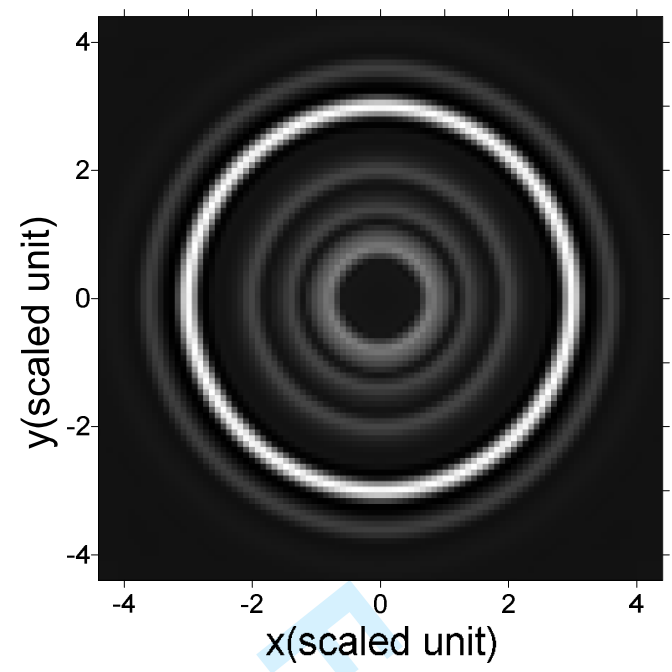

(a) $\varepsilon=-0.2$

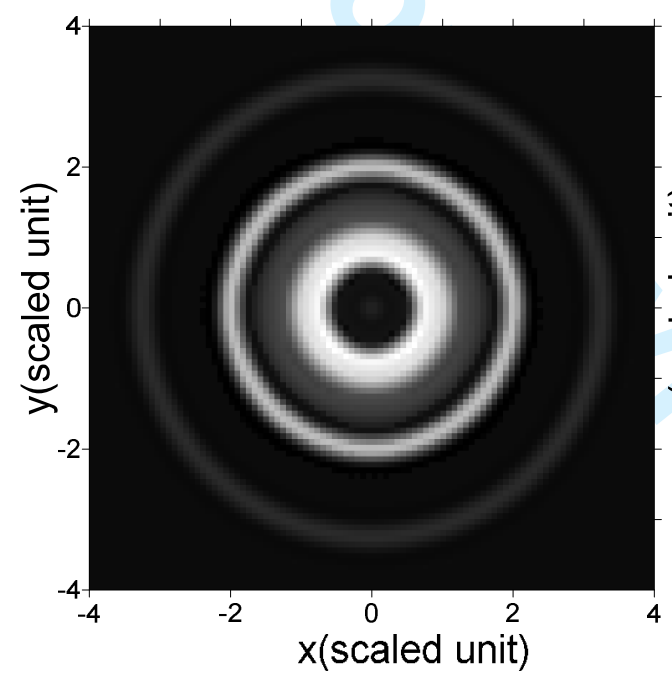

(c) $\varepsilon=-0.8$

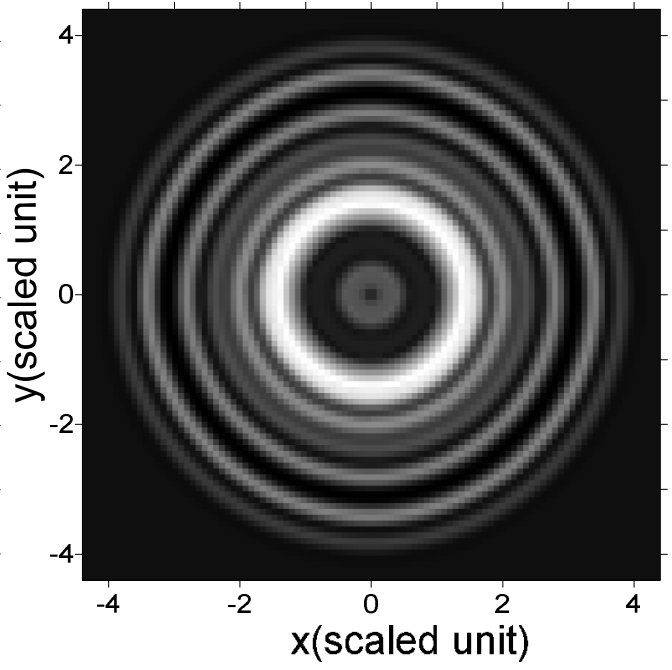

(b) $\varepsilon=-0.4$

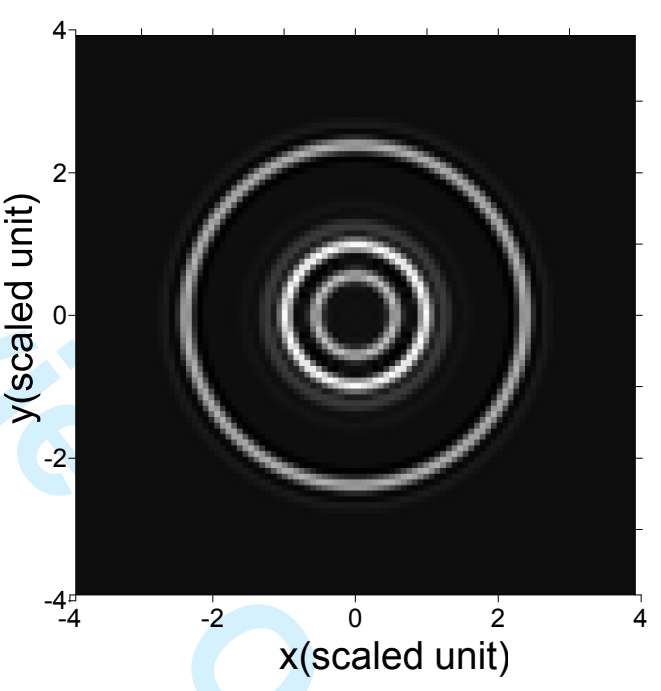

(d) $\varepsilon=-1.0$

Fig.8. The image plot of the electron probability density distribution on the detector plane at different scaled energies. The detector is located at $\mathrm{z}=-4.0$ plane. The scaled energy is given in each plot. 